This is consistent with the correlational data showing that dopamine neurons are activated not by reward per se, but by circumstances in which reward occurs unpredicted.

The authors also conducted several followup experiments to show that activation of the dopamine neurons could mitigate extinction of responding when the reward was reduced in value or omitted. This shows in a different context that activating dopamine neurons can prevent or retard learning. It is particularly relevant given a recent report that the some dopamine neurons increase firing to both unexpectedly good and bad events, possibly reflecting surprise in general or salience rather than the actual error signal reported more widely ${ }^{15}$. The effect on extinction demonstrated here suggests that, at least as a group, dopamine neurons are not signaling surprise or salience, as this would have been expected to enhance, rather than retard, learning.

As with any landmark study, the work of Steinberg and Janak ${ }^{4}$ opens the door to new questions as it closes the door on old ones. By showing that phasic activation of dopamine neurons is sufficient to replace a missing reward prediction error in such a wellcontrolled setting as blocking, this study allows us to now ask questions beyond this sticking point. For example it would be interesting to determine whether dopamine is also necessary for learning in a similar context. This could be readily done using unblocking. It would also be of interest to test whether learning unblocked by activation of the dopamine neurons reflects knowledge of the form and features of the specific reward that is present during the learning; this might be demonstrated by showing that responding to the unblocked cue is sensitive to devaluation of the reward or by showing that this cue can itself serve as a blocker. Such a demonstration would bolster the assertion that phasic dopamine acts as an error signal and not as a new reward.

Furthermore, it would be of interest to test more fully, using both causal and correlational approaches, whether the phasic dopamine signals have access to higher order information beyond what is contained in the present theoretical accounts mapped onto the firing of these neurons. Again, reliance on well-controlled learning theory tasks that force animals to rely on inference or model-based reasoning would provide a definitive answer. And finally, by combining these approaches with new molecular and genetic tools, it should be possible to also resolve questions about the specificity and possible heterogeneity of midbrain dopamine signals: whether the iconic, bidirectional reward prediction error is really all these neurons convey or whether the actual information represented in the release of dopamine (and perhaps co-release of other neurotransmitters in some areas) is more variable and perhaps targeted to different neural circuits. By resolving any real controversy over whether transient dopamine signals can replace missing reward prediction errors, the work by Steinberg and Janak ${ }^{4}$ provides a firm foundation from which such studies can proceed.

\section{COMPETING FINANCIAL INTERESTS}

The authors declare no competing financial interests.

1. Rescorla, R.A. \& Wagner, A.R. in Classical Conditioning II: Current Research and Theory (eds. Black, A.H. \& Prokasy, W.F.) 64-99 (Appleton-Century-Crofts, New York, 1972).

2. Pearce, J.M. \& Hall, G. Psychol. Rev. 87, 532-552 (1980).

3. Sutton, R.S. \& Barto, A.G. Psychol. Rev. 88, 135-170 (1981).

4. Steinberg, E.E. et al. Nat. Neurosci. 16, 966-973 (2013).

5. Schultz, W., Dayan, P. \& Montague, P.R. Science $\mathbf{2 7 5}$ 1593-1599 (1997).

6. Mirenowicz, J. \& Schultz, W. J. Neurophysiol. 72, 1024-1027 (1994).

7. Takahashi, Y.K. et al. Neuron 62, 269-280 (2009).

8. Iordanova, M.D., Westbrook, R.F. \& Killcross, A.S. Eur. J. Neurosci. 24, 3265-3270 (2006).

9. Darvas, M. \& Palmiter, R.D. Proc. Natl. Acad. Sci. USA 106, 14664-14669 (2009).

10. Tsai, H.C. et al. Science 324, 1080-1084 (2009).

11. Adamantidis, A.R. et al. J. Neurosci. 31 10829-10835 (2011).

12. Razran, G. Psychol. Bull. 63, 42-64 (1965).

13. Kamin, L.J. in Punishment and Aversive Behavior (eds. Campbell, B.A. \& Church, R.M.) 242-259 (Appleton-Century-Crofts, New York, 1969).

14. Waelti, P., Dickinson, A. \& Schultz, W. Nature 412 43-48 (2001).

15. Matsumoto, M. \& Hikosaka, O. Nature $459,837-841$ (2009).

\title{
Pair-bonding through epigenetics
}

Prairie voles (Microtus ochrogaster) are a socially monogamous species. Unlike other species of voles, they form long-lasting pair bonds with their partners. Studies comparing prairie voles to related species have revealed that oxytocin, vasopressin and dopamine signaling are necessary for the formation of partner preference. In males, partner preference requires vasopressin neurotransmission in the ventral pallidum and lateral septum, whereas oxytocin neurotransmission in the nucleus accumbens (NAc) and prelimbic cortex are essential in females. In both sexes, dopamine D2 receptors in the NAc facilitate the formation of partner preference, whereas dopamine D1 receptors inhibit pair-bonding. The longlasting nature of pair bonds suggests that equally enduring molecular mechanisms may underlie their formation. On page 919, Wang and colleagues show that histone modifications are involved in modifying gene expression to promote formation of partner preference.

The authors observed that mating, which normally induces partner preference in female prairie voles, led to increased expression of the oxytocin receptor (OTR) and vasopressin receptor (V1aR) in NAc of females. This increase in expression was associated with an increase in histone 3 acetylation

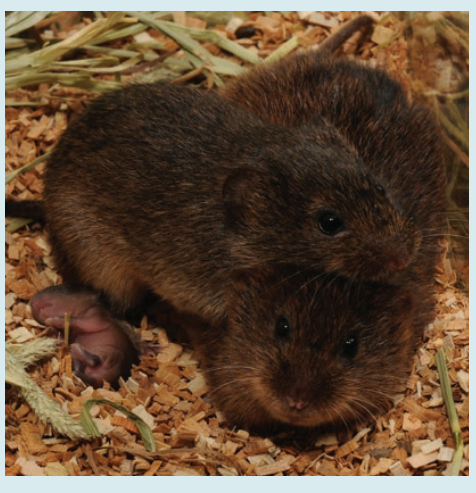
on lysine 14 (AcH3K14), a mark that is usually correlated with increased transcription, specifically at the oxtr and avprla promoters. If female voles were merely exposed to their prospective partners without mating, central administration of the histone deacetylase inhibitor trichostatin A (TSA) was sufficient to induce partner preference. Similar to mating, TSA infusion induced specific increases in AcH3K14 at the oxtr and avprla promoters and increased expression of OTR and V1aR in the NAc. Notably, infusion of OTR or V1aR antagonists into the brain blocked the ability of intra-NAc TSA to induce partner preference, indicating that these receptors are necessary for formation TSA-induced partner preference.

These results suggest that chromatin modifications underlie the formation of partner preference in prairie voles, adding to a growing list of behaviors that are regulated by such epigenetic mechanisms, which have previously been implicated in the formation of long-term memory, drug addiction and affective behaviors. Prairie voles, which exhibit more socially complex behaviors than many rodent species, have been championed as a model in which to study social affiliative behaviors more generally, and the hormones studied here, oxytocin and vasopressin, have been implicated in a wide range of social behaviors. These findings suggest that epigenetic mechanisms may also be involved in other social behaviors.

Brigitta Gundersen 